\title{
STUDY OF MULTIPARTICLE JET PRODUCTION USING CALORIMETERS, HIGH ENERGY CHANNELING, AND OTHER HIGH ENERGY PHYSICS RESEARCH
}

\section{Progress Report}

for Period April 1, 1990 - March 31, 1991
DOE/ER/ $02894--3$

DE9 1007362

Alvin S. Kanofsky

LEHIGH UNIVERSITY

Department of Physics

Bethlehem, PA 18015

October 1990

Prepared for

THE U.S. DEPARTMENT OF ENERGY AGREEMENT NO. DE-AC02-76ER02894

\section{NOT I C E}

\section{MASTER}

This report was prepared as an account of work sponsored by the United States Government. Neither the United States nor the Department of Energy, nor any of their employees, nor any of their contractors, subcontractors, or their employees, makes any warranty, express or implied, or assumes any legal liability or responsibility for the accuracy, completeness, or usefulness of any information, apparatus, product or process disclosed or represents that its use would not infringe privately-owned rights. 


\section{ABSTRACT}

During this period, work was started on the construction of the backward gamma chambers to be used in experiment E683 at Fermilab. Work also is proce ding on various auxiliary experiments that can be performed with the experimental set-up.

Work on the SSC accelerator and experiments began in 1984 with the participation of the principal investigator in the Snomass summer studies and continues with the funding of the accelerator and the location of its site in Texas. We are working with the large EMPACT/TEXAS collaboration on a large detector that the group has proposed. As well, the principal investigator is working on smaller experiments that can be executed at the SSC with less resources. Also, we are working on detector development and radiation tolerance of detectors such as scintillating fibers and data acquisition electronics.

Experiments continue at Lehigh on growing "whiskers" in wire chambers. These whiskers affect the forformance of wire chambers by providing corona discharge points within the chambers. We are able to study the growth of the whiskers in the laboratory and measure the whisker composition using Auger and ESCA spectroscopy and the scanning electron microscope.

We are further developing results that were obtained earlier from various Monte-Carlo programs, including the effects of various momentum energy conservation methods on results, extrapolations to higher transverse momentum, granularity of detectors, and resolution of previous discrepancies. A Ph.D. thesis was completed on this work.

Results of radiation effects on various electronic devices were obtained. Work continued on new accelerator devices and concepts. 
Progress on D.O.E. Research Contract \#DE-AC02-76-ER02984

April 1, 1991 - March 31, 1992

WIRE CHAMBER RECONSTRUCTION AND GAMMA DETECTOR FOR FERMILAB EXPERIMENT E683

Work was completed earlier on rebuilding the wire drift chambers for Fermilab experiment E683. These chambers were used in Fermilab experiment E609, but were no longer performing properly towards the end of the experimental running period. The Fermilab wire chamber winding facility was used to rewind the chambers. The chambers were tested with radioactive sources and in the beam line.

We also worked on improving the reliability of relays used in the calorimeter amplifiers. We developed an all electronic relay as well as studying how to improve the operation of the previously used mechanical relays.

We are presently working on the construction of a gamma ray backward scatter detector to be used with the E683 apparatus. This consists of $\mathrm{Pb}$ plates interspersed with wire proportional chambers and scintillating fibers in order to discriminate pi-zero's from single gamma rays. This will sit to one side of the target in the E683 experiment.

\section{WHISKER GROWTH STUDIES}

At Lehigh, we continued a program to study the growth of "whiskers" in wire chambers. We are able to grow the whiskers using pulsed voltages or continuous corona discharge. We can then measure their growth rate as a function of various parameters (gas composition, etc.) and we can analyze the structure and composition of the whiskers using Auger and ESCA spectroscopy or a Scanning Electron Microscope. We find that the deposits on the whiskers have a variety of elements, and we are able to obtain reasonable values for the composition using reaction kinetic models. Also, we studied the composition and structure of the whiskers that formed in the old E609 wire chambers.

\section{MONTE CARLO MODELS AND E609 RESULTS}

We have used various Monte-Carlo models to study the effects of the program parameters on the predicted results, and the comparison of these results with the existing experimental data. The two main programs that have been used are ISAJET and a program written at Argonne National Laboratory and used for the analysis of the E609 data. We have studied how various momentum-energy conservation methods affect the Monte-Carlo predictions and also how the generating transverse momentum 
range affects the results. Another area of study is the comparison of the results from the minimum bias events and the hard quark collision events and whether the data is best represented as a combination of the two. We have also studied how best to extrapolate the low transverse momentum results to higher momentum regions, and also the effects of calorimeter granularity and energy deposition approximation methods on the results. A Ph.D. thesis was completed earlier on this work.

Further analysis of the E609 data is continuing. The collaboration has studied the energy flow in hard proton-nucleus collisions at $400 \mathrm{GeV} / \mathrm{c}$. observed probability distribution of energy flow indicates that the energy transfer to a heavy nucleus seldom fluctuates to small values. We also find that the $A$ dependence of the di-jet production cross section, parameterized as $A^{b}$, steepens from a linear $A$ dependence for small values of energy flow in the target region to considerably larger values of $b$ as the energy flow to the target region increases.

As well, we have found new experimental information about the effect of a nucleus on partons which have undergone a hard scatter. We have observed clear dijet events from $400 \mathrm{GeV} / \mathrm{c}$ p-nucleus interactions and compared them to di-jets from p$p$ interactions. Our results show that the angular width of the individual jets is only slightly affected by the presence of the nucleus, but the acoplanarity of the jet pairs is sharply increased. Papers on these results are included in the appendix.

\section{RADIATION EFFECTS ON ELECTRO-OPTICAL DEVICES AND ELECTRONICS}

We continue to a study the effects of radiation on electro-optical devices such as LiNbO3 crystals and electronics devices e.g. HFET's, MESFET's , and GaAs laser driving circuits. We have exposed various devices to an electron beam at the Lehigh Van de Graaff. Doses beyond 3000 MRad have been used. The attenuation of light in the $\mathrm{LiNbO} 3$ waveguides has beer, measured and large attenuations have been measured at doses higher than 1 MRad. More recently, we have measured the switching frequency dependence of radiation damage. Some papers on these results are included in the appendix.

With LeCroy Corporation we are measuring the effects of radiation on high energy data acquisition electronics such as amplifiers and registers. The include the TRA1000 and TRA402 amplifiers, the MVL407 comparator, and the MVV200 and and the MLL400 registers. Papers on this are included in the appendix.

With the GaAs laser driving circuits, we have measured how the circuits fail with dosage. It is observed that above $100 \mathrm{Mrad}$, the circuits all fail for a variety of reasons. One of these is metallization of contacts on the circuits. Work is continuing on measurements to get a more fundamental understanding of the effects. As well, we have measured the transistor I vs. V curves for HFET and MESFET field effect transistors exposed to varying amounts of radiation. We can also measure the defect structure using DLTS. This work is in collaboration with and partially supported by the 
Naval Air Development Center at Warminster and Bell Laboratories, Allentown and Reading. Some papers on these results are included in the appendix.

\section{SSC ACTIVITIES AND ACCELERATOR DEVELOPMENT PROJECTS}

We are a member of the large EMPACT/TEXAS collaboration to develop a large detector for one of the intersection regions of the SSC. A copy of the introduction to this proposal is included in the appendix. We are also working on smaller experimients that can be performed with much less resources. Work is also proceeding on various acceierator development projects. We are studying RFQ's amd other devices. This work involves the development of the appropriate accelerator simulation programs - Poisson, Superfish, Parmtec, and their installation and running at Lehigh.

\section{TIME SPENT BY THE PRINCIPAL INVESTIGATOR ON CONTRACT ACTIVITIES.}

The Principal investigator spends $50 \%$ of his time during the year on high energy research covered by the contract. He supervises two technicians, undergraduates, and the $\mathrm{PhD}$ thesis work of two graduate students as well as directly participating in the research activity. 

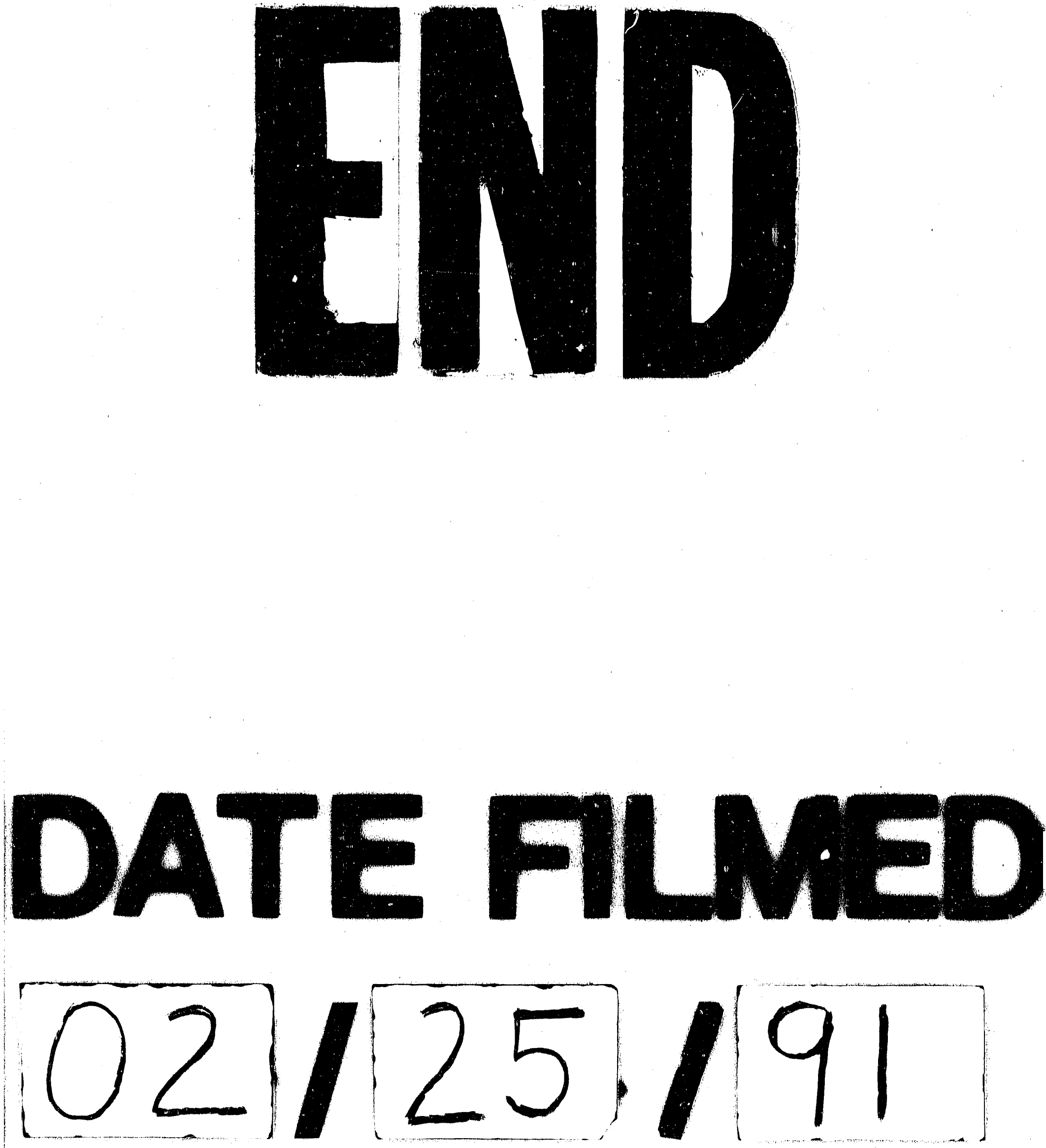
$\|, \quad, \quad, \cdot, \quad, \quad$, 